This Section of Epidemiology and Psychiatric Sciences appears in each issue of the Journal to stress the role of the epidemiological approach to promote advances in the field of clinical psychopharmacology, with a particular attention to controversial findings. The ultimate aims are to help develop a more critical attitude towards the results of research studies published in the international literature, to promote original research projects with higher methodological standards, and to implement the most relevant results of research in every-day clinical practice. These contributions are written in house by the journal's editorial team or commissioned by the Section Editor (no more than 1000 words, short unstructured abstract, 4 key-words, one Table or Figure and up to ten references).

Corrado Barbui, Section Editor

\title{
Antipsychotic combinations in schizophrenia
}

\author{
C. Gastaldon*, D. Papola and G. Ostuzzi \\ Department of Neuroscience, Biomedicine and Movement Sciences, Section of Psychiatry, University of Verona, Verona, Italy
}

\begin{abstract}
In the treatment of resistant schizophrenia, a number of meta-analyses attempted to quantify the efficacy and tolerability of antipsychotic (AP) polypharmacy $v$. monotherapy with contradictory results. Recently, a systematic review and metaanalysis of randomised controlled trials investigated the efficacy and tolerability of AP combination $v$. monotherapy in schizophrenia. It included 31 studies: 21 double-blind (considered high-quality studies) and 10 open-label (considered low-quality studies). The meta-analysis showed that, overall, the combination of two APs was more effective than monotherapy in terms of symptom reduction (standardised mean difference (SMD) $=-0.53,95 \%$ confidence interval (CI) -0.87 to -0.19 ); however, this result was confirmed only in the subgroup of low-quality studies. Negative symptoms improved when combining a D2 antagonist with a D2 partial agonist (SMD $=-0.41,95 \% \mathrm{CI}-0.79$ to -0.03 ) both in double-blind and open-label studies. In the present commentary, the results of this systematic review are critically discussed in terms of their clinical and research implications.
\end{abstract}

Received 27 March 2017; Accepted 4 May 2017; First published online 5 June 2017

Key words: Antipsychotics, evidence-based psychiatry, randomised controlled trials, schizophrenia, systematic reviews.

Despite some advances, the pharmacological treatment of schizophrenia continues to be a challenge (Kane \& Correll, 2010), especially in poor treatment responders, who represent a difficult to treat population with low rates of recovery that have not increased over time (Jaaskelainen et al. 2013).

In patients with poor response to antipsychotics (AP), current evidence-based guidelines recommend a range of different strategies, such as waiting for a delayed response, checking adherence, dose adjustment, switching to another AP. If these strategies are ineffective and at least two trials of different APs, prescribed at adequate dose and length of treatment, have failed to provide substantial benefit, patients are

* Address for correspondence: Dr C. Gastaldon, Section of Psychiatry, University of Verona, Ospedale Policlinico GB Rossi, Piazzale L.A. Scuro, 10 - 37134 Verona, Italy.

(Email: chiara.gastaldon@gmail.com) considered treatment-resistant (Suzuki et al. 2011, 2012). In treatment-resistant cases, guidelines recommend clozapine and, only if this attempt has failed, the combination of clozapine with a second AP (Lehman et al. 2004; NICE, 2009). Although guidelines suggest this combination strategy in selected circumstances only, in clinical practice combining two APs is quite common (Gallego et al. 2012), ranging between 10 and $30 \%$ of those receiving AP treatment (Correll \& Gallego, 2012).

The evidence in support of AP combination has been summarised by a number of systematic reviews that attempted to quantify the beneficial and harmful effects of this strategy, but so far results have been rather contradictory, with low confidence in overall treatment estimates. Probably, the inclusion of studies with small sample sizes, heterogeneity in study quality and the inclusion of studies where a second AP is added to a first one together with studies where two AP drugs 
are simultaneously prescribed from the beginning of treatment, are possible explanations for such heterogeneity and uncertainty (Barbui et al. 2009; Correll et al. 2009; Sommer et al. 2012; Taylor et al. 2012).

In order to overcome these limitations, Galling et al recently conducted a systematic review and meta-analysis focused on the efficacy and tolerability of AP combination strategies $v$. monotherapy in schizophrenia (Galling et al. 2017). A total of 31 randomised controlled trials (RCTs) with 2073 participants were included, and studies were grouped and analysed according to quality. RCTs that employed an open-label design and an 'observed-case' analysis were defined as low-quality, while RCTs that employed a double-blind approach and carried out an 'intention-to-treat' analysis were defined as highquality. In double-blind studies, the addition of a second $\mathrm{AP}$ to current $\mathrm{AP}$ treatment was compared with the addition of placebo, whereas in open-label studies, the addition of a second AP was compared with AP continuation in monotherapy. Pragmatically, therefore, the review included only trials in which monotherapy was compared with the addition of a second AP, excluding trials in which patients started treatment with two APs from the beginning. This choice was made to resemble ordinary practice as much as possible.

Efficacy analyses included only RCTs that used efficacy as the primary outcome (22 studies; $N=1342 ; 13$ high-quality and 9 low-quality studies). A number of efficacy measures were analysed, including symptoms reduction; response; reduction of positive, negative and depressive symptoms; reduction in the Clinical Global Impression Scale-Improvement (CGI-I) and functioning. AP combination was superior to monotherapy in terms of total symptoms reduction (16 studies, $N=694$, standardised mean difference (SMD) $=-$ $0.53,95 \%$ confidence interval (CI) -0.87 to -0.19 ) and response rate. However, subgroup analysis showed that this finding was confirmed in low-quality studies (seven studies, $N=316, \mathrm{SMD}=-0.83,95 \% \mathrm{CI}$ -1.16 to -0.50 ), but not in high-quality studies (nine studies, $N=378, \mathrm{SMD}=-0.30,95 \% \mathrm{CI}-0.78$ to 0.19 ). Overall, clozapine in combination with other APs was superior to clozapine alone $(\mathrm{SMD}=-0.52,95 \%$ CI -0.90 to -0.14 ), but again this was confirmed only in the subgroup of low-quality studies and not in high-quality studies. No advantage of any specific $\mathrm{AP}$ combination was shown, and this also applied to clozapine, in line with findings from previous reviews (Barbui et al. 2009).

Meta-regression analysis found that when converting AP doses into chlorpromazine equivalents, in comparison with standard AP dose, high AP dose was associated with less symptom improvement. Also, higher baseline symptom severity was associated with less symptom improvement, and this result was confirmed in the subgroup of high-quality studies.

Interestingly, negative symptoms, which are clinically perceived as particularly resistant to treatment, significantly improved when combining a D2 antagonist with a D2 partial agonist (eight studies, $N=532$, $\mathrm{SMD}=-0.41,95 \% \mathrm{CI}-0.79$ to -0.03$)$, even though there were only four high-quality trials confirming this result in subgroup analysis $(\mathrm{SMD}=-0.28,95 \%$ CI -0.55 to -0.009 ).

Discontinuation due to any cause and due to tolerability issues did not differ between AP combination and monotherapy. However, AP combination was associated with less insomnia and higher prolactin levels, while AP combination with aripiprazole was associated with reduced prolactin plasma levels and weight, confirming previous findings (Li et al. 2013). As a general limitation, few studies reported data about side effects (9 of 31).

The authors suggested that the gap between the results of double-blind and open-label studies might be explained by the so-called salience bias (Galling et al. 2017), which means that, when the allocation is known, effectiveness may be strongly influenced by clinicians' and patients' expectations. In particular, there is evidence that lack of double blindness is associated with inflated estimates of treatment effect, and this is particularly important when response criteria are subjective, as it happens with rating scale scores (Schulz et al. 1995). If investigators are not blinded, their attitudes for or against an intervention may be directly transferred to participants (Wolf, 1950), may enhance or reduce the overall intensity of their support/assistance to patients (performance bias) and may lead to differential assessment of outcomes (information or ascertainment bias) (Schulz \& Grimes, 2002). Moreover, if participants are not blinded, knowledge of group assignment can affect responses to the intervention received (Schulz et al. 2002). This is particularly relevant in this case, as combining two medications is generally perceived as more effective than monotherapy both by clinicians and patients.

Overall, the interpretation of this review is limited by the heterogeneity of trials, which inevitably included populations of patients with different characteristics. For example, the choice of including both clozapine and non-clozapine combination trials might have led to the inclusion of two different types of population, as in clinical practice prescription of clozapine is limited to treatment-resistant patients (Gillespie et al. 2017). Although translating evidence into recommendations is a sophisticated process that involves different stages, the results of this review, in the context of previous evidence summaries, suggest that clinicians should: 
- Avoid starting polypharmacy as a first step, since increased effectiveness is uncertain, while poor adherence, difficulties in the overall treatment management (Kane et al. 2013), interactions with other drugs and increased risk of medical unwanted events (Oderda et al. 2012; Barbui et al. 2014; Hsu et al. 2015; Nosè et al. 2015; Yu et al. 2016) are all expected negative consequences of polypharmacy;

- Use combination of two APs only after trials with at least two APs in monotherapy have been adequately conducted (in terms of dose and length of treatment) (Suzuki et al. 2012) and after an adequate trial with clozapine in monotherapy (Lehman et al. 2004; Barbui et al. 2009; NICE, 2009);

- The choice of an AP combination strategy should be made taking into consideration the characteristics of individual patients, carefully assessing comorbidities (e.g. heart disease, metabolic syndrome) and concurrent medical treatments, aiming to prescribe the less harmful AP (Leucht et al. 2013). If weight gain or increased prolactin is expected to be a challenging issue, or if negative symptoms are a clinical problem, then aripiprazole may be added (Arango et al. 2004);

- When prescribing two AP concurrently, the overall AP dose should be carefully considered, as most AP adverse effects are dose-dependent (Ray et al. 2009; Barbui et al. 2016);

- All choices should be made as part of a shared decision-making process that involves patients, family members, carers and other persons that may have a formal or informal role in the treatment programme.

This meta-analysis has important implications for research, as it pointed out that further studies with a focus on side effects and tolerability are needed. The relationship between combination of APs with D2 partial agonists and negative symptoms also should be investigated by means of trials comparing combination to switching strategies, helping better understand whether the effect is due to a specific effect of D2 partial agonists (Galling et al. 2017).

In conclusion, current evidence on patients with poor response to AP monotherapy is still weak, and large, pragmatic, high-quality trials are needed to better support clinicians and patients in making clinical decisions on this compelling issue.

\section{Financial support}

None.

\section{Conflict of interest}

None.

\section{References}

Arango C, Buchanan RW, Kirkpatrick B, Carpenter WT (2004). The deficit syndrome in schizophrenia: implications for the treatment of negative symptoms. European Psychiatry 19, 21-26.

Barbui C, Signoretti A, Mule`S, Boso M, Cipriani A (2009). Does the addition of a second antipsychotic drug improve clozapine treatment? Schizophrenia Bulletin 35, 458-468.

Barbui C, Conti V, Cipriani A (2014). Antipsychotic drug exposure and risk of venous thromboembolism: a systematic review and meta-analysis of observational studies. Drug Safety 37, 79-90.

Barbui C, Bighelli I, Carrà G, Castellazzi M, Lucii C, Martinotti G, Nosè M, Ostuzzi G, Star Network Investigators (2016) Antipsychotic dose mediates the association between polypharmacy and corrected QT interval. PLOS ONE 11, e01 48212.

Correll CU, Gallego JA (2012). Antipsychotic polypharmacy: a comprehensive evaluation of relevant correlates of a long-standing clinical practice. Psychiatric Clinics of North America 35, 661-681.

Correll CU, Rummel-Kluge C, Corves C, Kane JM, Leucht S (2009). Antipsychotic combinations vs monotherapy in schizophrenia: a meta-analysis of randomized controlled trials. Schizophrenia Bulletin 35, 443-457.

Gallego JA, Bonetti J, Zhang J, Kane JM, Correll CU (2012). Prevalence and correlates of antipsychotic polypharmacy: a systematic review and meta-regression of global and regional trends from the 1970s to 2009. Schizophrenia Research 138, 18-28.

Galling B, Roldán A, Hagi K, Rietschel L, Walyzada F, Zheng W, Cao XL, Xiang YT, Zink M, Kane JM, Nielsen M, Leucht S, Correll CU (2017). Antipsychotic augmentation vs. monotherapy in schizophrenia: systematic review, meta-analysis and meta-regression analysis. World Psychiatry 16, 77-89.

Gillespie AL, Samanaite R, Mill J, Egerton A, MacCabe JH (2017). Is treatment-resistant schizophrenia categorically distinct from treatment-responsive schizophrenia? A systematic review. BMC Psychiatry 17, 12.

Hsu W, Esmaily-Fard A, Lee C (2015). Antipsychotic exposure and risk of stroke: a systematic review and meta-analysis of observational studies. Value in Health 18, A828.

Jaaskelainen E, Juola P, Hirvonen N, McGrath JJ, Saha S, Isohanni M, Veijola J, Miettunen J (2013). A systematic review and meta-analysis of recovery in schizophrenia. Schizophrenia Bulletin 39, 1296-1306.

Kane JM, Correll CU (2010). Past and present progress in the pharmacologic treatment of schizophrenia. Journal of Clinical Psychiatry 71, 1115-1124.

Kane JM, Kishimoto T, Correll CU (2013). Non-adherence to medication in patients with psychotic disorders: epidemiology, contributing factors and management strategies. World Psychiatry 12, 216-226.

Lehman AF, Lieberman JA, Dixon LB, McGlashan TH, Miller AL, Perkins DO, Kreyenbuhl J, American Psychiatric Association, Steering Committee on Practice Guidelines (2004). Practice guideline for the treatment of patients with schizophrenia, second edition. American Journal of Psychiatry 161(2 Suppl.), 1-56. 
Leucht S, Cipriani A, Spineli L, Mavridis D, Orey D, Richter F, Samara M, Barbui C, Engel RR, Geddes JR, Kissling W, Stapf MP, Lässig B, Salanti G, Davis JM (2013).

Comparative efficacy and tolerability of 15 antipsychotic drugs in schizophrenia: a multiple-treatments meta-analysis. Lancet 382, 951-962.

Li X, Tang Y, Wang C (2013). Adjunctive aripiprazole versus placebo for antipsychotic-induced hyperprolactinemia: meta-analysis of randomized controlled trials. PLOS ONE 8, e70179.

NICE, National Institute for Health and Clinical Excellence (2009). Schizophrenia: Core Interventions in the Treatment and Management of Schizophrenia in Adults in Primary and Secondary Care (Update). http://www.nice.org.uk.

Nosè M, Recla E, Trifiro G, Barbui C (2015). Antipsychotic drug exposure and risk of pneumonia: a systematic review and meta-analysis of observational studies.

Pharmacoepidemiology and Drug Safety 24, 812-820.

Oderda LH, Young JR, Asche CV, Pepper GA (2012). Psychotropic-related hip fractures: meta-analysis of first-generation and second-generation antidepressant and antipsychotic drugs. Annals of Pharmacotherapy 46, 917-928.

Ray WA, Chung CP, Murray KT, Hall K, Stein CM (2009). Atypical antipsychotic drugs and the risk of sudden cardiac death. New England Journal of Medicine 360, 225-235.

Schulz KF, Grimes DA (2002). Blinding in randomised trials: hiding who got what. Lancet 359, 696-700.

Schulz KF, Chalmers I, Hayes RJ, Altman DG (1995).

Empirical evidence of bias. Dimensions of methodological quality associated with estimates of treatment effects in controlled trials. JAMA 273, 408-412.

Schulz KF, Chalmers I, Altman DG (2002). The landscape and lexicon of blinding in randomized trials. Annals of Internal Medicine 136, 254-259.

Sommer IE, Begemann MJH, Temmerman A, Leucht S (2012). Pharmacological augmentation strategies for schizophrenia patients with insufficient response to clozapine: a quantitative literature review. Schizophrenia Bulletin 38, 1003-1011.

Suzuki T, Remington G, Mulsant BH, Rajji TK, Uchida H, Graff-Guerrero A, Mamo DC (2011). Treatment resistant schizophrenia and response to antipsychotics: a review. Schizophrenia Research 133, 54-62.

Suzuki T, Remington G, Mulsant BH, Uchida H, Rajji TK, Graff-Guerrero A, Mimura M, Mamo DC (2012). Defining treatment-resistant schizophrenia and response to antipsychotics: a review and recommendation. Psychiatry Research 197, 1-6.

Taylor DM, Smith L, Gee SH, Nielsen J (2012). Augmentation of clozapine with a second antipsychotic - a meta-analysis. Acta Psychiatrica Scandinavica 125, 15-24.

Wolf S (1950). Effects of suggestion and conditioning on action of chemical agents in human subjects: pharmacology of placebos. Journal of Clinical Investigation 29, 100-109.

Yu Z, Jiang H, Shao L, Zhou Y, Shi H, Ruan B (2016). Use of antipsychotics and risk of myocardial infarction: a systematic review and meta-analysis. British Journal of Clinical Pharmacology, 1-9. 